$326 \pm 342$, and $400 \pm 240$, respectively ( $\mathrm{p}=0.001)$. The higher NK cells levels were significantly associated with the survival of the patients in the follow-up time ( $\mathrm{p}=0.009)$.

Conclusion: Pre-chemotherapy NK cell levels failed to demonstrate a significant predictor of response to chemotherapy. However, the higher pre-chemotherapy NK cell levels are associated with favorable patient follow-up outcome. This study is only a preliminary report of NK cell in Thai gynecologic cancer. A well-design and larger population study may be further conducted to clarify the role of this immunologic factor.

Poster (M05)

Quality of Life, Nursing \& Supportive Care

https://doi.org/10.3802/jgo.2021.32.S1.M05

\section{Perspective and conception of Thai gynecologic oncologists in palliative care}

Panida Mathaveechotikul," Chuenkamon Charakorn, Arb-aroon Lertkhachonsuk, Navamol Lekskul, Sarikapan Wilailak, Kittiphon Nagaviroj

Ramathibodi Hospital, Bangkok, Thailand (Panidamtv@gmail.com)

Objective: To evaluate the conception and perspective on palliative care (PC) in Thai gynecologic oncologists.

Methods: The online survey was distributed to all certificated Thai gynecologic oncologists. The survey could be accessed via working email address, hyperlink, or QR code during May 2020 and January 2021. A 5-point Likert scale captured the perspectives and concepts of PC. The association between respondents' characteristics and their choices of content in PC, together with their decision making in specified clinical scenarios was analyzed. Results: A total of 207 completed surveys from 320 Thai gynecologic oncologists were received (64.6\% participation rate). They prospected a willingness to give the advices to both patients and their families (85.5\%), and strongly agreed to introduce PC in any stage of cancer at the time of diagnosis $(75.8 \%)$. The numbers of their palliative cases per year were 5-20 (57.97\%) and the PC teams were available in their hospitals. They decided to offer early PC and do-not-resuscitate (DNR), especially for the elders, or patients with advance stages, or recurrent disease. We found that gynecologic oncologists who previously experienced a PC training did not show any difference in decision making in specified clinical scenarios, compared with who did not.

Conclusion: Thai gynecologic oncologists responded to the conceptions and perspectives in PC. Their concepts of early and willingness to offer a PC especially in the elders, advanced stage, or recurrent patients were proven, regardless of the experience in PC training.
Poster (M06)

Quality of Life, Nursing \& Supportive Care

https://doi.org/10.3802/jgo.2021.32.S1.M06

\section{Prevalence of malnourishment and predictive factors associated with the nutritional status of gynecologic cancer patients undergoing chemotherapy: a cross-sectional analysis}

\author{
Khemmanat Sanguanwongthong, Prapaporn Suprasert \\ Chiang Mai University, Chiang Mai, Thailand (psuprase@gmail.com)
}

Objective: To investigate the prevalence of malnourishment and predictive factors for nutritional status of gynecologic cancer patients treated with chemotherapy.

Methods: One hundred and one gynecologic cancer patients treated with chemotherapy between April 2020 and February 2021 were interviewed for Patient Generated-Subjective Global Assessment (PG-SGA). Clinical data including body mass index (BMI), underlying disease, the diagnosis, FIGO staging, the recurrent status, the surgical intervention, the performance status, the chemotherapy detail, the total number of lymphocytes per cubic milliliter (TLC) and serum albumin were collected. Levels of TLC and serum albumin were calculated for an optimal cut-off point by using receiver operating characteristic curves. The clinicopathological variables were compared using univariate and multivariate analysis to identify the independent predictive factors for malnourishment status. Results: The prevalence of well, moderate and severe nourishment were $73.3 \%, 18.8 \%$ and $7.9 \%$, respectively. The optimal cut-off points for TLC was 1,450 cells/ $\mu \mathrm{L}$ and for albumin was $3.9 \mathrm{~g} / \mathrm{dL}$. The univariate analysis indicated that the number of present chemotherapy $<3$ cycles, album in level $<3.95 \mathrm{~g} / \mathrm{dL}, \mathrm{BMI}<25 \mathrm{~kg} / \mathrm{m}^{2} \mathrm{TLC}<1,450$ cells $/ \mu \mathrm{L}$, anemia and no neutropenia were significant associated factors for malnourishment. However, only serum albumin $<3.95 \mathrm{~g} / \mathrm{dL}$ was independent associated factor for malnourishment (adjusted odds ratios $=6.709 ; 95 \%$ confidence interval $=2.113-21.304$ ) . Conclusion: Nearly one-fourth of patients with gynecologic cancer who on going treatment with chemotherapy were found malnourishment. The only independent associated factor related to the nutritional status was serum albumin $<3.95 \mathrm{~g} / \mathrm{dL}$.

Poster (M07)

Quality of Life, Nursing \& Supportive Care https://doi.org/10.3802/jgo.2021.32.S1.M07

\section{Validity of the Comprehensive Score for Financial Toxicity (COST) tool in gynecologic cancer patients}

